\title{
FDG-PET hypermetabolism is associated with higher tau-PET in mild cognitive impairment at low amyloid-PET levels
}

Anna Rubinski ${ }^{1}$, Nicolai Franzmeier ${ }^{1}$, Julia Neitzel ${ }^{1}$, Michael Ewers ${ }^{1,2^{*}}$ (D) and the Alzheimer's Disease Neuroimaging Initiative (ADNI)

\begin{abstract}
Background: FDG-PET hypermetabolism can be observed in mild cognitive impairment (MCl), but the link to primary pathologies of Alzheimer's diseases (AD) including amyloid and tau is unclear.

Methods: Using voxel-based regression, we assessed local interactions between amyloid- and tau-PET on spatially matched FDG-PET in $72 \mathrm{MCl}$ patients. Control groups included cerebrospinal fluid biomarker characterized cognitively normal $(C N, n=70)$ and $A D$ dementia subjects $(n=95)$.

Results: In MCl, significant amyloid-PET by tau-PET interactions were found in frontal, lateral temporal, and posterior parietal regions, where higher local tau-PET was associated with higher spatially corresponding FDG-PET at low levels of local amyloid-PET. FDG-PET in brain regions with a significant local amyloid- by tau-PET interaction was higher compared to that in $\mathrm{CN}$ and $\mathrm{AD}$ dementia and associated with lower episodic memory.

Conclusion: Higher tau-PET in the presence of low amyloid-PET is associated with abnormally increased glucose metabolism that is accompanied by episodic memory impairment.
\end{abstract}

Keywords: FDG-PET, Hypermetabolism, Tau-PET, Amyloid-PET, Hyperactivation, Mild cognitive impairment

\section{Introduction}

In Alzheimer's disease (AD), alterations in glucose metabolism as assessed by $\left[{ }^{18} \mathrm{~F}\right]$ fluorodeoxyglucose positron emission tomography (FDG-PET) are a common pathological hallmark [1]. Specifically, FDG-PET hypometabolism within temporoparietal regions is commonly observed in AD dementia and earlier AD stages, including in amyloid-positive mild cognitive impairment (MCI; i.e., prodromal $\mathrm{AD})[2]$ and cognitively normal $(\mathrm{CN})$ elderly at genetic risk of AD [3]. However, FDG-PET metabolism shows complex changes during the course of

\footnotetext{
* Correspondence: Michael.Ewers@med.uni-muenchen.de

${ }^{1}$ Institute for Stroke and Dementia Research, Klinikum der Universität

München, Ludwig-Maximilians-Universität LMU, Feodor-Lynen-Straße 17, 81377 Munich, Germany

${ }^{2}$ German Center for Neurodegenerative Diseases, Munich, Germany
}

$\mathrm{AD}$, where not only reductions but also increases in FDG-PET metabolism have been reported across $\mathrm{CN}$ amyloid-positive subjects [4] and subjects at genetic risk of $\mathrm{AD}[5,6]$ and $\mathrm{MCI}$ [7]. Thus, clinical staging of cognitive symptoms does not correspond to FDG-PET alterations in a straightforward manner.

Studies using amyloid- and tau-PET imaging suggest that these pathologies are important predictors of regional FDG-PET alterations. For amyloid-PET, elevated global levels of amyloid-PET have been associated with reduced FDG-PET in both AD dementia [8] and MCI [9]. However, increased FDG-PET has also been observed in association with elevated amyloid-PET [4]. Furthermore, there is a poor regional match between amyloid-PET and FDG-PET in typical [10] and atypical AD [11] suggesting that amyloid-PET alone cannot fully 
account for FDG-PET alterations. Results from tau-PET studies suggest that tau pathology may be an important modulating factor of FDG-PET [12-14]. Results from recent studies in elderly asymptomatic $\mathrm{CN}$ revealed an interaction between amyloid- and tau-PET, where higher tau-PET was associated with higher FDG-PET at low levels of amyloid-PET, but with lower levels of FDGPET at high levels of amyloid-PET $[15,16]$. These results provide an intriguing model of the dynamic bidirectional changes in relationship to beta-amyloid $(A \beta)$ and tau pathology. The focus on biomarkers of $A \beta$ and tau pathology rather than the clinical diagnosis of $\mathrm{AD}$ allows to investigate the effect of different mixtures of both pathologies on FDG-PET changes and cognitive impairment. This is important because even in the absence of abnormal levels of $A \beta$, abnormal tau-PET levels can be observed in higher cortical brain areas in a substantial number of elderly subjects, where higher tau-PET was associated with cognitive impairment [17]. However, the association of higher tau-PET with FDG-PET alterations at varying levels of $A \beta$ in symptomatic elderly subjects is unclear. In order to address this research gap, we examined both the main and interaction effects of $\left[{ }^{18} \mathrm{~F}\right] \mathrm{AV} 45$ amyloid-PET and $\left[{ }^{18} \mathrm{~F}\right] \mathrm{AV} 1451$ tau-PET on FDG-PET in subjects with amnestic MCI. Furthermore, we tested whether the observed higher levels of FDG-PET represent abnormally increased FDG-PET, i.e., FDG-PET hypermetabolism, and whether such increases in FDG-PET are beneficial or detrimental for cognition.

\section{Methods}

\section{Participants}

All subjects were recruited within the Alzheimer's Disease Neuroimaging Initiative (ADNI phase III; http:// adni.loni.usc.edu/) [18]. Inclusion criteria for the current study beyond those of ADNI were a diagnosis of MCI at the PET acquisition visit (Mini-Mental State Examination $(\mathrm{MMSE})>24$, Clinical Dementia Rating $(\mathrm{CDR})=$ 0.5 , objective memory loss on the education-adjusted Wechsler Memory Scale II, preserved activities of daily living) and the availability of $\left[{ }^{18} \mathrm{~F}\right] \mathrm{AV} 1451$ tau-PET, $\left[{ }^{18} \mathrm{~F}\right] \mathrm{AV} 45$ amyloid-PET, and $\left[{ }^{18} \mathrm{~F}\right]$ FDG-PET up to 6 months apart. From the total sample of $74 \mathrm{MCI}$ subjects fulfilling the inclusion criteria, two subjects failed preprocessing and were excluded, yielding a final sample of $72 \mathrm{MCI}$ subjects. Apolipoprotein E (APOE) genotyping was available as well.

In addition to the MCI group with all three PET modalities, a group of 70 cerebrospinal fluid (CSF) $A \beta$ - and p-tau 181 -negative $\mathrm{CN}$ subjects (MMSE $>24, \mathrm{CDR}=0$ ) and $95 \mathrm{AD}$ dementia subjects (MMSE $<26, \mathrm{CDR}>0.5$, fulfillment of NINCDS/ADRDA criteria for probable AD) [19] were also included to assess group-level differences in regional FDG measures. These subjects were recruited in ADNI phase II and were selected for the current study based on the availability of FDG-PET and CSF biomarkers of $A \beta$ and tau. $C N$ subjects were asymptomatic and $A \beta$ and phosphorylated tau ( $\mathrm{p}$-tau) negative based on a quantitative CSF threshold (Elecsys CSF immunoassay; $\mathrm{A} \beta_{1-42}>976.6 \mathrm{pg} / \mathrm{ml}, \quad \mathrm{p}$-tau ${ }_{181}<21.8 \mathrm{pg} / \mathrm{ml}$ [20];). AD dementia subjects were diagnosed based on ADNI diagnostic criteria and were CSF biomarker positive (Elecsys CSF immunoassay; $\mathrm{A} \beta_{1-42}<976.6 \mathrm{pg} / \mathrm{ml}$, p$\left.\operatorname{tau}_{181}>21.8 \mathrm{pg} / \mathrm{ml}[20]\right)$.

\section{MRI and PET acquisition}

All MRI data were obtained on 3-T scanner systems at each ADNI site according to standardized protocol. Tau-PET data were acquired for 30-min dynamic emission scan, six 5-min frames, 75-105 min post-injection of $10.0 \mathrm{mCi}$ of $\left[{ }^{18} \mathrm{~F}\right] \mathrm{AV} 1451$. Amyloid-PET data were acquired for 20-min dynamic emission scan, four 5-min frames, $50-70 \mathrm{~min}$ post-injection of $10.0 \mathrm{mCi}$ of $\left[{ }^{18} \mathrm{~F}\right]$ AV45. FDG-PET data were acquired for 30-min dynamic emission scan, six 5-min frames, 30-60 min postinjection of $5.0 \mathrm{mCi}$ of $\left[{ }^{18} \mathrm{~F}\right] \mathrm{FDG}$. PET data underwent extensive quality control protocols and standardized image preprocessing correction steps to produce uniform data across the ADNI centers. These steps included frame co-registration, averaging across the dynamic range, and standardization with respect to the orientation, voxel size, and intensity [21]. Detailed information on the imaging protocols and standardized image preprocessing steps for MRI and PET can be found at http://adni.loni.usc.edu/methods.

\section{MRI and PET preprocessing}

T1 MRI images acquired in closest temporal proximity to the tau-PET scan were preprocessed using the same SPM12-based (Wellcome Trust Centre for Neuroimaging, University College London) pipeline as described previously [18]. Briefly, for each subject, the T1 MRI image was segmented into gray matter (GM), white matter (WM), and CSF maps. Next, non-linear highdimensional spatial normalization parameters were estimated, and a group-specific template was created using SPM's DARTEL toolbox. The group-specific template was linearly registered to the MNI template in order to estimate the affine transformation parameters.

For each subject, tau-PET, amyloid-PET, and FDGPET images were coregistered to the participant's T1 MRI image in native space. For the voxel-based analyses, all PET images were subsequently spatially warped to MNI space using the DARTEL flow fields and affine transformation parameters estimated based on the MRI spatial registration described above. For all PET modalities, standardized uptake value ratio (SUVR) images were computed using the inferior cerebellar gray for tau- 
PET, the whole cerebellum for amyloid-PET, or the pons for FDG-PET as reference regions. A GM mask was created by warping the group-average GM map from the DARTEL template to MNI space and binarizing the image to only include voxels that had at least $30 \%$ GM probability. We further excluded subcortical structures (basal ganglia, thalamus, cerebellum, and brain stem) from the mask because they were either used as reference region or in order to avoid inclusion of regions that show off-target $\left[{ }^{18} \mathrm{~F}\right] \mathrm{AV} 1451$ binding likely unrelated to tau [22]. All PET images were GM masked and smoothed using an 8-mm Gaussian smoothing kernel.

\section{Creation of $z$-transformed deviation images (z-maps)}

To assess differences in tau deposition, we computed voxel-wise mean and standard deviation of SUVR values for $\mathrm{CN}$. The $\mathrm{CN}$ group was recruited in ADNI phase III and consisted of 27 amyloid-negative $\mathrm{CN}$ subjects with $\left[{ }^{18} \mathrm{~F}\right] \mathrm{AV} 1451$ tau-PET. $z$-score deviation maps were created for each of the MCI subjects, by subtracting from each voxel the voxel-wise mean and dividing by the standard deviation of CN group SUVR.

\section{Assessment of amyloid status}

Amyloid status was computed using a pre-established protocol [23]. Specifically, T1 MRI images were segmented and parcellated into cortical regions with Freesurfer (v5.3; surfer. nmr.mgh.harvard.edu/), which was used to extract mean amyloid-PET uptake from GM regions (frontal, lateral temporal, lateral parietal, and anterior/posterior cingulate) relative to the whole cerebellum. Participants were classified as amyloid-positive or amyloid-negative based on established cut-points (global amyloid-PET SUVR $\geq 1.11$ ) [23].

\section{Cognitive assessment}

To estimate memory performance, we used ADNIMEM, an episodic memory composite score based on a broad battery of neuropsychological memory tests [24]. The ADNI-MEM score includes the Rey Auditory Verbal Learning Test, the Alzheimer's Disease Assessment Scale, the Wechsler Logical Memory I and II, and the word recall of the MMSE.

\section{Statistical analysis}

Demographics were compared between diagnostic groups using $t$ tests for continuous variables and chisquared tests for categorical variables.

We conducted voxel-based linear regression analyses to test the main effect as well as the local interactions amyloid- by tau-PET on FDG-PET. All analyses were controlled for age, gender, education, study site, and - in case of testing the interaction effect - the main effects of amyloid- and tau-PET. All PET measures were included as continuous variables and obtained in spatially corresponding voxels across all three PET modalities, thus assessing the local relationship between the variables. These calculations were done via the software package VoxelStats, a MATLAB (Mathworks Inc., Natick, MA, USA)-based package for multimodal voxelwise brain image analysis [25]. The customized GM mask (see above) was used to constrain the analysis to cortical GM. The voxel-based statistical parametric maps were corrected for multiple comparisons, where the statistical significance was defined using a random field theory-based [26] threshold of $p<0.05$ with a cluster forming threshold of $p<0.001$. In order to examine the nature of the amyloid- by tau-PET interaction, significant voxel clusters of the interactions were identified and labeled according to the largest overlap to the automated anatomical labeling regions. For all three PET modalities, we extracted the mean voxel values within each cluster showing significant amyloid- by tau-PET interactions on FDG-PET resulting from the voxel-wise analyses. We plotted the interactions to ensure that results were not driven by extreme values. The robustness of the interaction effect for each cluster was tested by rerunning the regression model after removing influential cases defined by Cook's distance $D$ [27]. Observations with large influence (the threshold for considering an observation as influential was defined as $4 /$ number of observations) and observations exceeding 3 standard deviations from the mean were excluded in order to test whether the regression coefficient remained significant. Clusters were considered significant and stable when meeting an alpha threshold of 0.05 after removing influential cases.

In addition, post hoc interaction analyses on the mean cluster values were conducted controlling additionally for APOE genotype status (APOE $\varepsilon 4$ allele carriers vs non-carriers).

Group-level differences in regional FDG measures were assessed by a one-way ANCOVA (controlling for age, gender, education, and study site) with post hoc $t$ test between each pair to assess the difference between MCI subgroups and control groups.

In order to test whether FDG-PET cluster values were associated with memory performance, we conducted for each cluster a linear regression analysis including ADNIMEM scores as the dependent variable and the FDGPET cluster values as the predictor, controlling for age, gender, education, and study site.

All statistical analyses were performed using R-statistical software (http://www.R-project.org). Associations were considered significant when meeting an alpha threshold of 0.05 .

\section{Results}

Sample characteristics

Demographic characteristics and group differences are presented in Table 1. Figure 1 shows the tau-PET 
Table 1 Group characteristics (mean \pm SD)

\begin{tabular}{llll}
\hline & CN $(\boldsymbol{n}=\mathbf{7 0})$ & $\mathbf{M C l}(\boldsymbol{n}=\mathbf{7 2})$ & AD dementia $(\boldsymbol{n}=\mathbf{9 5})$ \\
\hline Age (years) & $72.00 \pm 5.48^{\mathrm{C}}$ & $76.74 \pm 7.33$ & $74.11 \pm 8.60^{\mathrm{a}}$ \\
Gender (M/F) & $33 / 37$ & $42 / 30$ & $52 / 44$ \\
Education (years) & $16.53 \pm 2.65$ & $16.33 \pm 2.88$ & $15.48 \pm 2.68$ \\
MMSE & $28.99 \pm 1.22^{\mathrm{b}}$ & $28.22 \pm 1.88$ & $22.98 \pm 2.04^{\mathrm{c}}$ \\
$\mathrm{A} \beta-/ \mathrm{A} \beta+^{\mathrm{d}}$ & $70 / 0$ & $42 / 30$ & $0 / 95$
\end{tabular}

Abbreviations: $A \beta$ amyloid-beta, $A D$ Alzheimer's disease, $C N$ cognitively normal, $F$ female, $M$ male, $M C l$ mild cognitive impairment, MMSE Mini-Mental State Exam

Significantly different from $\mathrm{MCl}-{ }^{\mathrm{a}} p<0.05,{ }^{\mathrm{b}} p<0.01$, and ${ }^{\mathrm{C}} p<0.001$

${ }^{\mathrm{d}} \mathrm{A} \beta$ status was determined via PET in $\mathrm{MCl}$ and via $\mathrm{CSF}$ in $\mathrm{CN}$ and $\mathrm{AD}$ dementia groups

distribution within amyloid-negative $\mathrm{CN}$ subjects. TauPET levels predominantly in the temporal lobe were higher in MCI compared to those in amyloid-negative $\mathrm{CN}$ (Fig. 1b).

\section{Voxel-wise amyloid- and tau-PET main effects on FDG-PET metabolism}

First, we tested the main effects of amyloid- and tauPET on FDG-PET in MCI. As shown in Fig. 2 (for statistics, see supplementary Table 1), higher amyloid-PET was associated with higher FDG-PET in small clusters located in the right superior frontal, right occipital, left cuneus, and right temporal pole. On the other hand, higher tau-PET was associated with higher FDG-PET in multiple regions within the bilateral parietal lobe, left insular, and cingulate cortices. Negative associations were primarily observed within the left middle frontal and left temporoparietal regions.

When stratified by amyloid status (global amyloidPET SUVR $\geq 1.11$ ), the associations between higher
tau-PET and higher FDG-PET metabolism are evident only within the amyloid-negative subgroup, while the opposite association was primarily observed in the amyloid-positive subgroup (Fig. 2, Table 1).

\section{Voxel-wise amyloid- by tau-PET interactions on FDG-PET metabolism}

Since we found that the associations between tau-PET and FDG-PET are dependent on A $\beta$ levels, we further tested the local amyloid- by tau-PET interaction on FDG-PET in MCI. Linear regression analysis of the interaction of amyloid-PET by tau-PET (included as continuous variables) showed significant effects in multiple brain regions. In order to examine whether any outliers may drive these interactions, we extracted the mean voxel values in each cluster and examined the undue influence of any observations based on Cook's distance. Those clusters that survived the quality check are displayed in Fig. 3a (for statistics, see Table 2).

All amyloid-PET by tau-PET interactions were of the same direction, i.e., higher tau-PET was associated with higher FDG-PET at low levels of amyloid-PET but not at high levels of amyloid-PET (Fig. 3b). These clusters were predominantly located within the left middle temporal gyrus, right inferior temporal gyrus, right lingual gyrus, left precuneus, bilateral inferior parietal gyrus, left superior frontal gyrus, and right middle frontal gyrus.

To determine whether these effects were driven by differences in $A P O E$ status, we tested whether $A P O E$ status had influenced the results. When controlling all above listed models for $A P O E$, the observed interactions remained significant $(p<0.05)$ in all clusters.

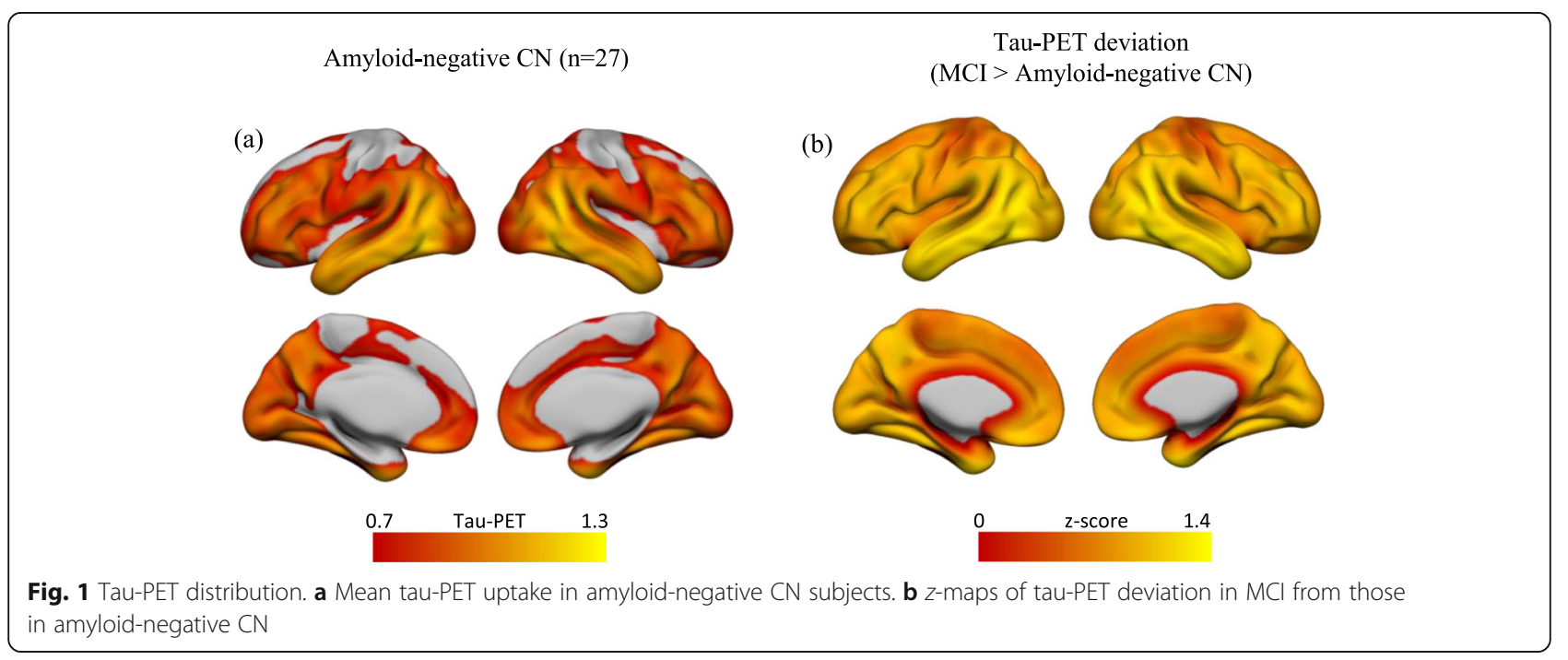




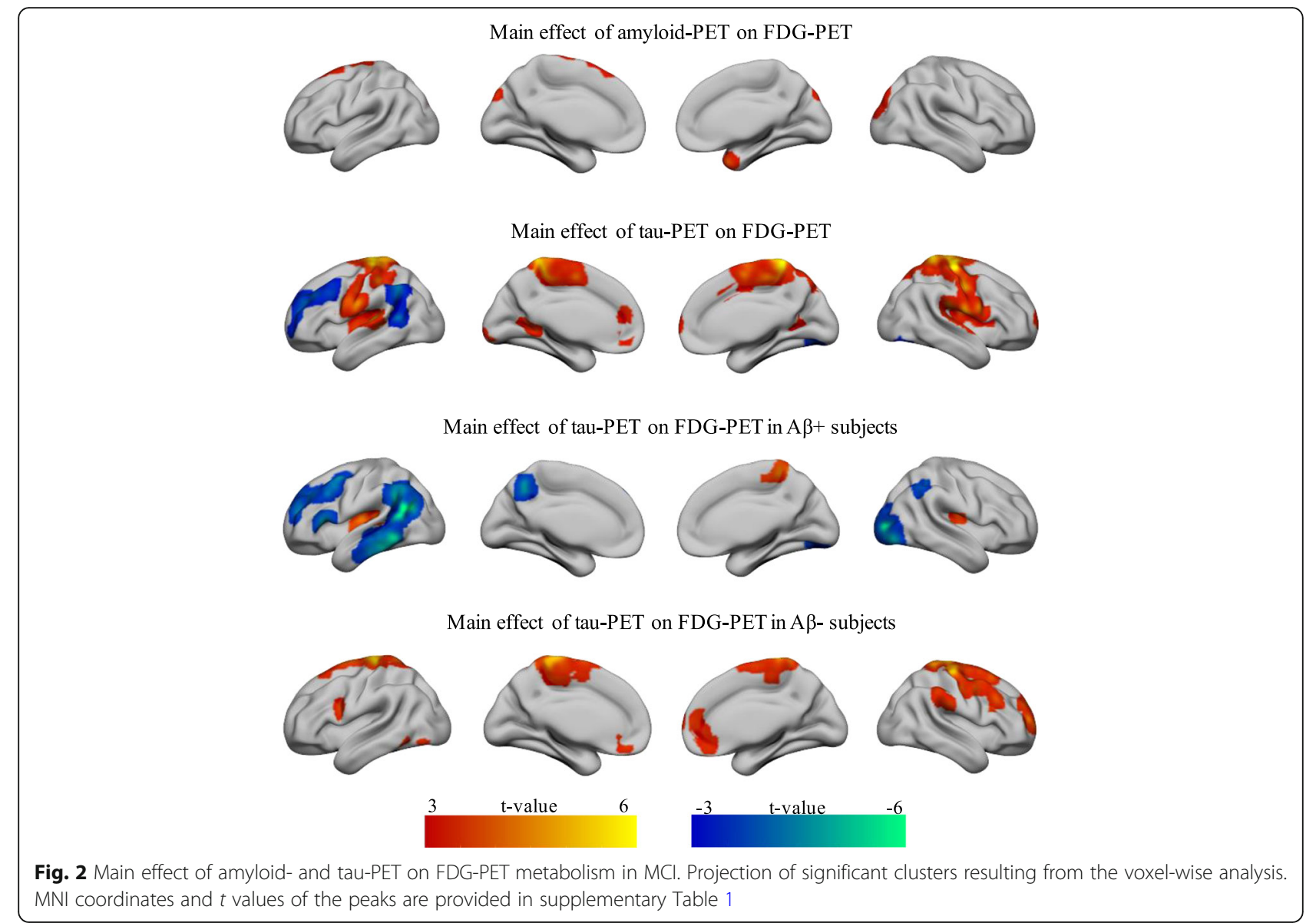

Tau-related hypermetabolism in amyloid-negative $\mathrm{MCI}$ subjects

In order to examine whether the observed tau-related increase in FDG-PET cluster values in the MCI subjects with low amyloid represented abnormal FDG-PET hypermetabolism, we compared the FDG-PET cluster values in the MCI subgroups to the FDG-PET in amyloid-negative $\mathrm{CN}(n=70)$ and subjects with fullblown AD dementia $(n=95)$. Note that these two reference groups including $\mathrm{CN}$ and $\mathrm{AD}$ dementia were characterized by CSF biomarker profile of $\mathrm{A} \beta_{1-42}$ and p$\mathrm{tau}_{181}$ rather than amyloid- and tau-PET given that those imaging modalities were not available in a sufficiently large number of $\mathrm{CN}$ and $\mathrm{AD}$ dementia subjects.

MCI subjects were divided by high and low tau-PET (median split) and by amyloid status (global amyloidPET SUVR $\geq 1.11$ ), resulting in four subgroups (high vs low tau/positive vs negative amyloid). FDG-PET levels for all MCI subgroups along with the control groups are plotted in Fig. 4. ANCOVA showed significant $(p<0.05)$ group differences in FDG-PET for all clusters except for one cluster within the left superior frontal gyrus $(p=$
0.067). Post hoc analyses confirmed that the tau-related increase in FDG-PET in the high-tau/amyloid-negative MCI subgroup was significantly higher compared to the $\mathrm{CN}$ group in clusters located within the right middle frontal, left middle temporal, and right lingual gyri. The same group also had significantly higher FDG-PET levels compared to $\mathrm{AD}$ dementia cases within the same clusters, confirming that the FDG-PET levels will eventually decrease with clinical $\mathrm{AD}$ progression.

\section{Hypermetabolism in the right middle frontal cortex is associated with lower memory performance}

Next, we addressed the question whether tau-related FDG-PET hypermetabolism in MCI is associated with memory performance. Since FDG-PET hypermetabolism was observed at lower levels of amyloid-PET (see above), we chose to test FDG-PET cluster values as predictors of memory performance in amyloid-negative MCI subjects in each cluster. We found a significant association in the right middle frontal ( $p=0.013$; Fig. 5$)$. The association was negative, meaning higher FDG-PET metabolism in the middle frontal gyrus cluster of FDG-PET 
(a)
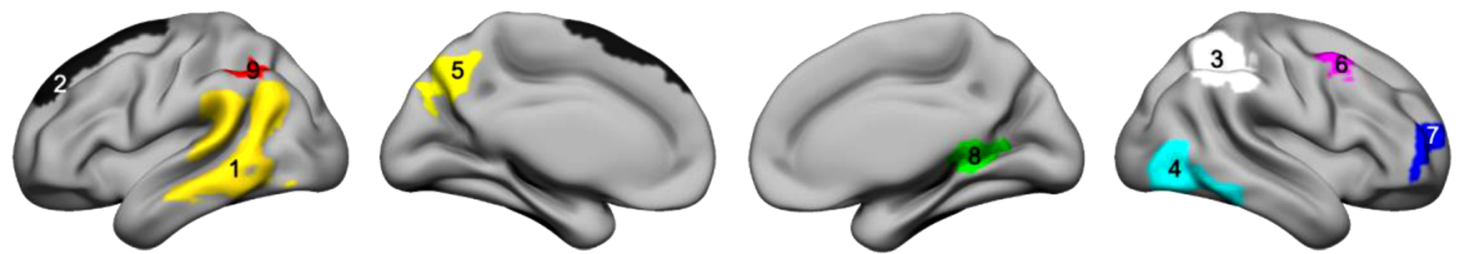

(b)
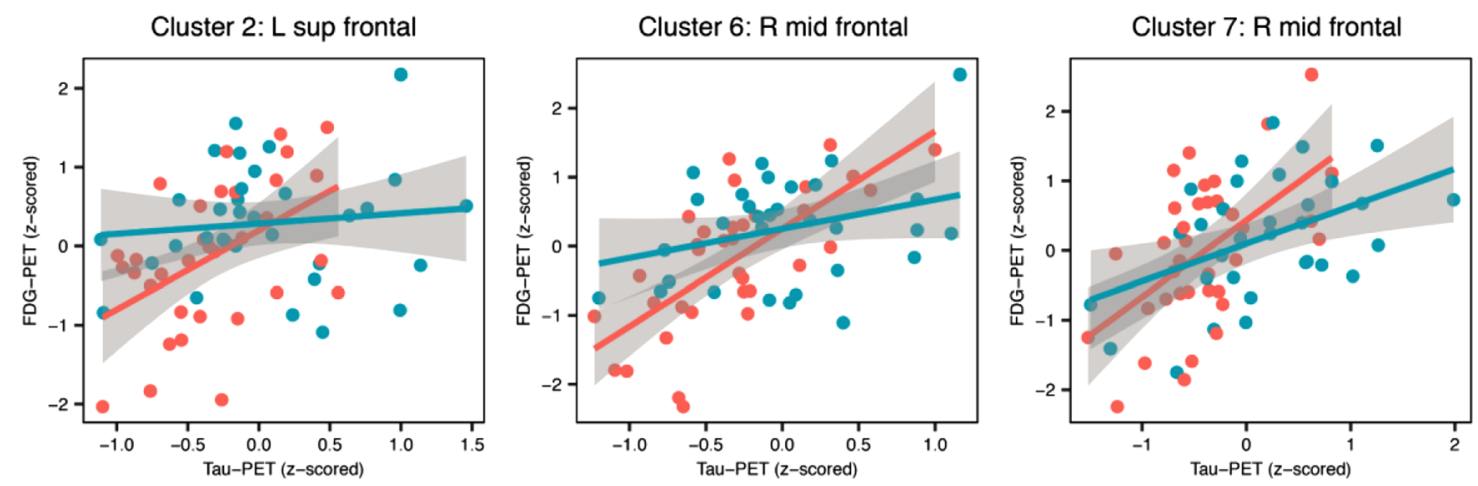

Amyloid-PET

- High

- Low

Cluster 3: R inf parietal
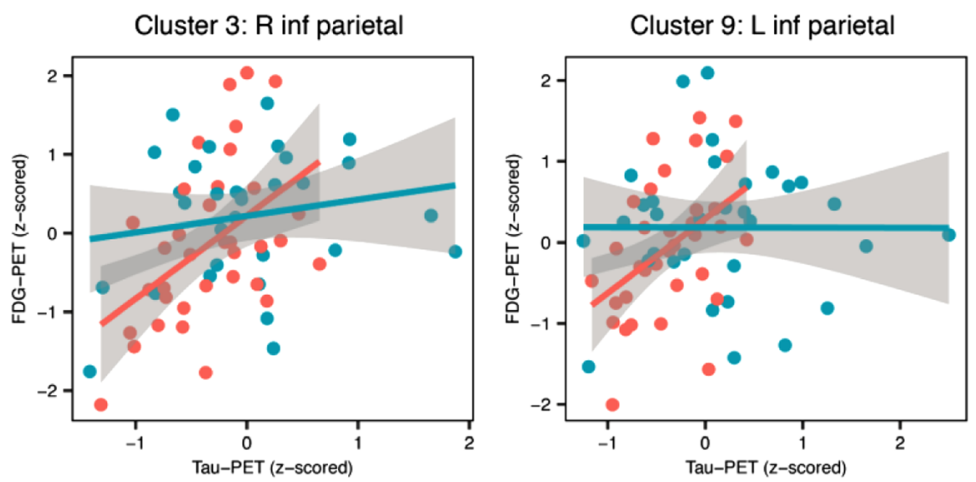

Cluster 5: L precuneus

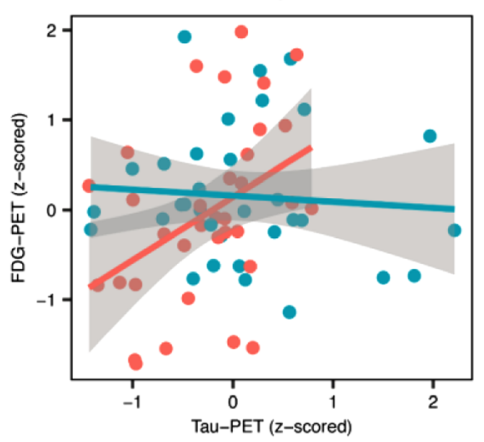

Cluster 1: L mid temporal
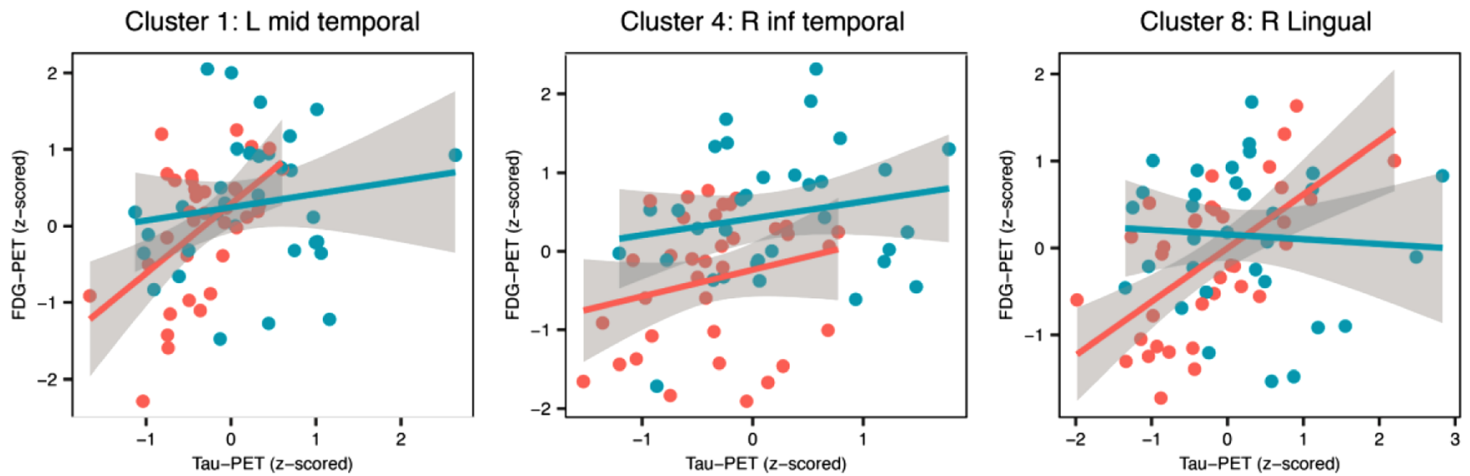

Fig. 3 Regional interactions between amyloid- and tau-PET on FDG-PET metabolism in MCl. a Projection of significant clusters resulting from the voxel-wise analysis. b Scatterplots are based on mean SUVR values extracted from voxel-wise analyses for each of the significant clusters (arranged by anatomical adjacency). For all statistical analyses, amyloid-PET was used as a continuous measure; for illustrational purposes, however, amyloid levels were binarized into high and low levels (median split). Scatterplots are presented after removal of outliers (i.e., defined as influential observations by Cook's distance and 3 standard deviations from the mean); for regression plots including the outliers, see supplementary Fig. 1 
Table 2 Areas showing significant voxel-wise interaction between amyloid- and tau-PET on FDG-PET in $\mathrm{MCl}$

\begin{tabular}{lllllll}
\hline Labels & $\begin{array}{l}\text { Cluster } \\
\text { index }\end{array}$ & $\begin{array}{l}\text { Size } \\
\text { (voxels) }\end{array}$ & \begin{tabular}{l}
$\boldsymbol{t}$ value \\
\cline { 5 - 8 }
\end{tabular} & & \multicolumn{2}{c}{ MNI coordinates } \\
\hline L middle temporal & 1 & 3552 & 5.64 & -51 & -60 & 24 \\
L superior frontal & 2 & 1853 & 6.4 & -7.5 & 27 & 63 \\
R inferior parietal & 3 & 1448 & 6.11 & 52.5 & -36 & 51 \\
R inferior temporal & 4 & 1122 & 5.29 & 58.5 & -49.5 & -24 \\
L precuneus & 5 & 606 & 5.91 & -4.5 & -76.5 & 39 \\
R middle frontal & 6 & 444 & 6.24 & 37.5 & 10.5 & 61.5 \\
R middle frontal & 7 & 432 & 5.07 & 36 & 57 & 13.5 \\
R lingual & 8 & 224 & 4.39 & 21 & -48 & 6 \\
L inferior parietal & 9 & 191 & 4.25 & -45 & -51 & 52.5 \\
\hline
\end{tabular}

$L$ left, $R$ right

$\mathrm{MNI}$ coordinates and $t$ values of the peaks are provided. $t$ values are based on voxel-wise regressions controlling for age, gender, education, and study site.

hypermetabolism was associated with a lower ADNIMEM score. This result suggests that right frontal FDGPET hypermetabolism is associated with worse memory performance. Control analysis in the amyloid-positive MCI subjects did not show significant associations between FDG-PET and cognition for any of the clusters.

\section{Discussion}

Our first major finding showed that higher tau-PET was associated with higher glucose metabolism in subjects with lower levels of amyloid-PET, but not higher levels of amyloid-PET. These effects were predominantly found within the middle temporal gyrus, posterior parietal, and frontal cortex and were independent of APOE genotype. Our second major finding was that the taurelated increases in FDG-PET represented hypermetabolism since the FDG-PET level exceeded that of $\mathrm{CN}$ and $\mathrm{AD}$ dementia subjects. Our third major finding was that the tau-related FDG-PET hypermetabolism in MCI subjects with low amyloid was associated with lower memory performance.

Our findings advance the current understanding of FDG-PET changes in MCI, providing an explanatory model of FDG-PET hypermetabolism that has been observed in multiple studies in asymptomatic and symptomatic elderly subjects (for a review, see [28]). In line with our results, a recent study in MCI reported increased FDG-PET metabolism at low levels of amyloidPET but not high levels of amyloid-PET [7]. FDG-PET metabolism was positively associated with $A \beta$ in $\mathrm{MCI}$, but inversely associated with $A \beta$ in $A D$ dementia [29]. We show that tau-PET plays an important role in FDGPET hypermetabolism in MCI subjects at low $A \beta$ levels, suggesting the interaction of tau and amyloid pathology in non-demented subjects to be key for the increase in
FDG-PET. Compared to the interaction approach, our analysis of tau-PET stratified by negative vs positive amyloid-PET showed a more widespread association of higher tau-PET and FDG-PET. Higher tau-PET was preferentially associated with higher FDG-PET in $A \beta$ negative MCI subjects, but with lower FDG-PET in A $\beta$ positive subjects, consistent with the results of our interaction analyses. The spatially more restricted interaction effect is probably due to lower statistical power to test an interaction effect compared to testing a main effect.

Our results are consistent with recent findings in $\mathrm{CN}$, where higher tau-PET was associated with higher FDGPET in participants with low levels of amyloid-PET [15, 16]. We expand significantly above those previous results by showing that the interaction extends to MCI, where the tau-related increase in FDG-PET represents hypermetabolism above normal levels and is associated with lower memory performance. These findings on FDG-PET show parallels to fMRI detected hyperactivation as a function of tau and amyloid pathology. Both resting-state and task-evoked hyperactivity, especially in the medial temporal lobe [30], but also other brain regions [31] has been observed in early-phase autosomal dominant AD [32] and MCI [30, 31, 33]. fMRI-assessed hyperactivation in the medial temporal lobe was associated with faster cognitive decline in MCI [33], consistent with our findings of FDG-PET hypermetabolism to be associated with lower cognitive performance in MCI. Furthermore, fMRI-assessed hyperactivation was associated with higher tau-PET in $\mathrm{CN}[34,35]$. An interaction of tau-PET by amyloid-PET on resting-state fMRIassessed network connectivity in $\mathrm{CN}$ was observed, such that after a phase of hyperconnectivity, there was a decline in network connectivity when both tau-PET and amyloid-PET were high [36]. These results are reminiscent of the interaction effect of tau-PET by amyloid-PET on FDG-PET observed in the current study. Together, these studies suggest a synergistic interaction of tau and amyloid pathology on brain activity assessed across different modalities.

In the current study, we took a biomarker-centered approach using amyloid- and tau-PET to predict changes in FDG-PET in MCI. A subset of the MCI patients showed no abnormal A $\beta$ levels. Higher tau-PET levels in the absence of abnormal $A \beta$ levels may be due to primary age-related tauopathy (PART) [37]. PART is characterized by elevated tau pathologies confined to Braak-stage regions I-IV at absent or low levels of amyloid plaques and has been proposed to be an etiological entity that is qualitatively different from $\mathrm{AD}[37,38]$. Although it is still debated whether PART is part of the $\mathrm{AD}$ continuum [39], it is generally accepted that abnormal $A \beta$ levels are a defining feature of AD. Thus, 


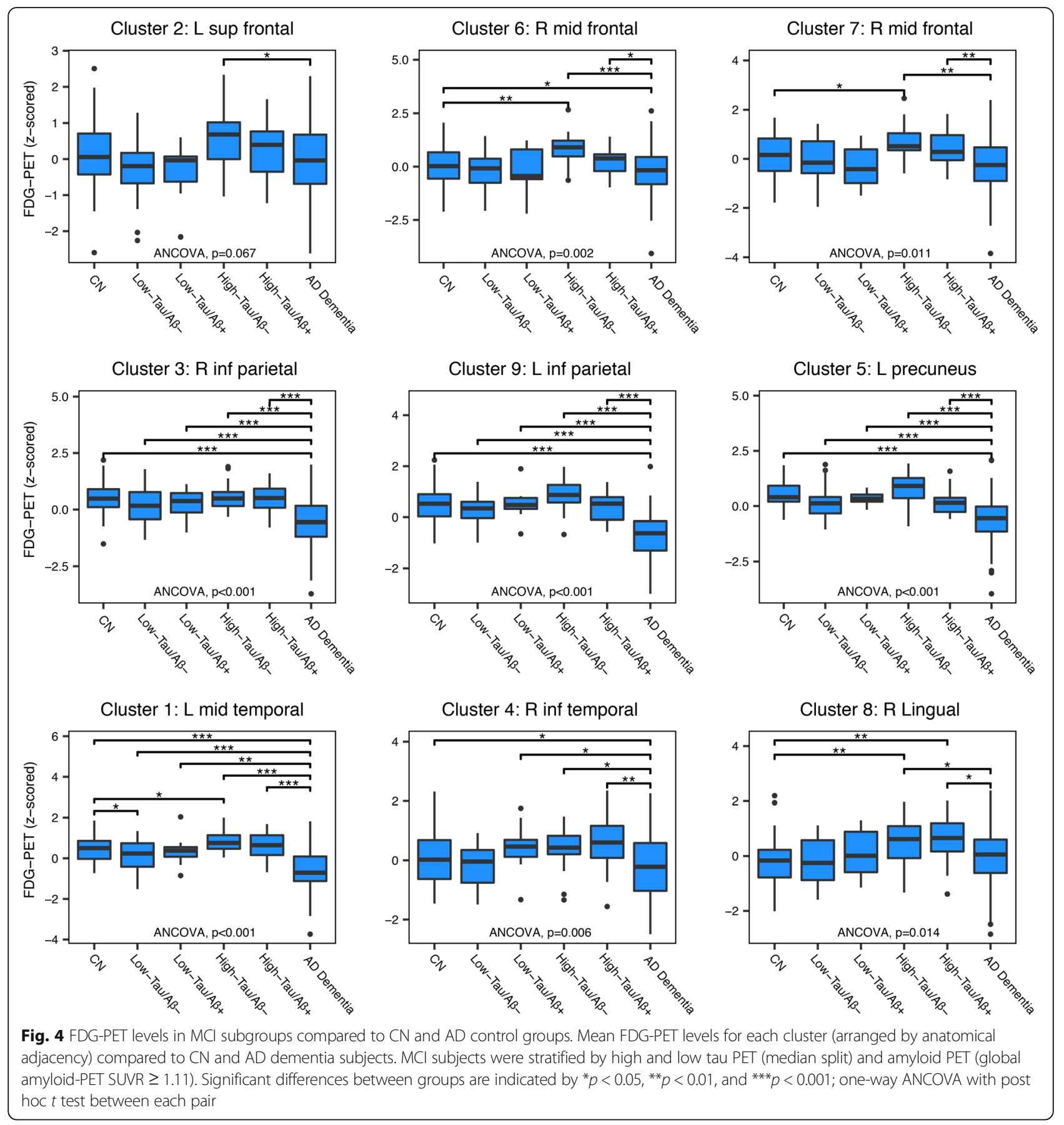

not all MCI participants were within the AD continuum. Nevertheless, based on biomarker-driven rather than diagnostic characterization, our study showed that the interaction between both types of $\mathrm{AD}$ pathologies is predictive of FDG-PET alterations.

The mechanism by which pathologic tau or amyloid is associated with an increase in glucose metabolism remains an open question. In vitro electrophysiological analysis showed that secreted extracellular tau fragments obtained post-mortem from the brain of an individual with $\mathrm{AD}$ cause neuronal hyperactivity in human neurons [40]. Moreover, transgenic mice studies showed that reducing tau protein levels in the brain is associated with reduced susceptibility to neuronal hyperexcitability and seizures [41], suggesting that tau modulates neuronal hyperactivity of neuronal networks [42]. The disruption 


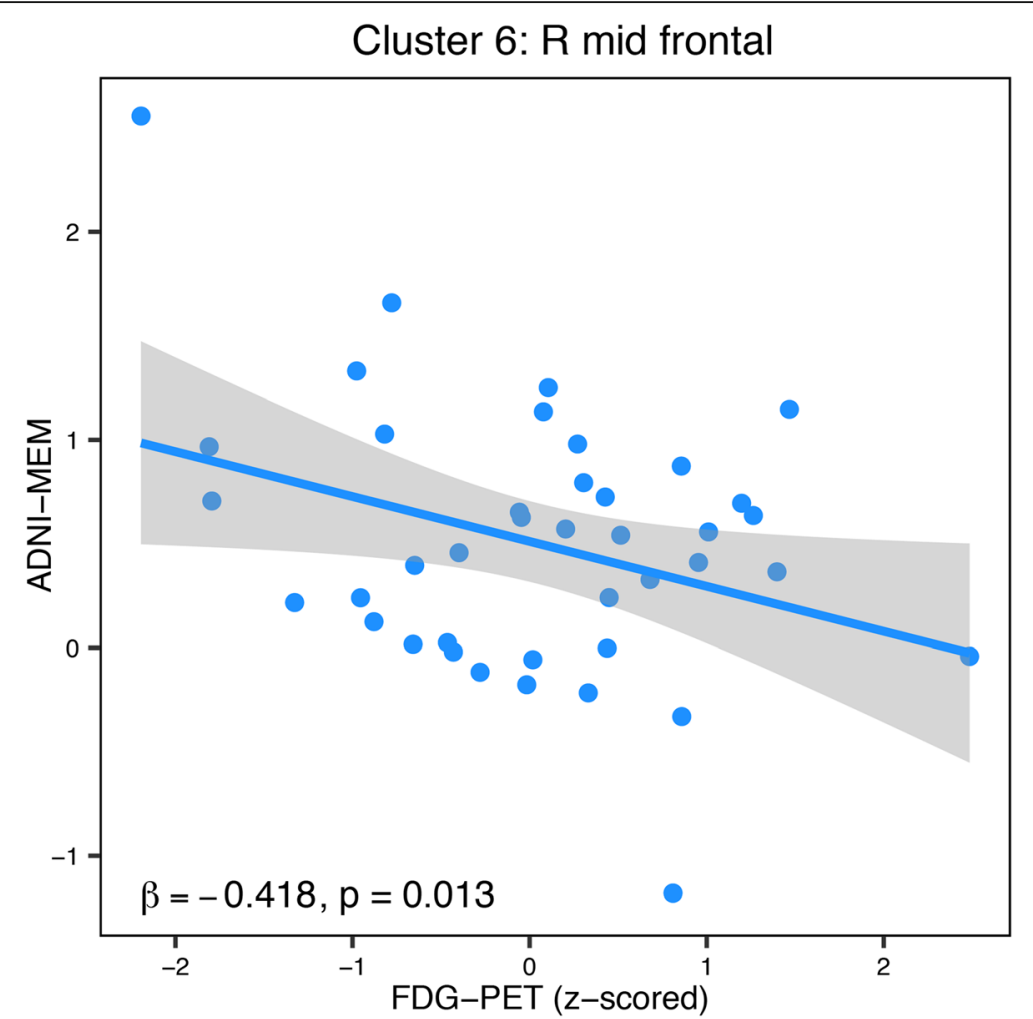

Fig. 5 Associations among FDG-PET and memory performance. Scatterplot for the regression model of FDG-PET on ADNI-MEM in amyloid-negative $\mathrm{MCl}$ subjects

of GABAergic neuronal network has been suggested as a possible mechanism of tau-associated disturbance of hippocampal neuron excitability [43]. The differential role of tau and amyloid in driving hypermetabolism is somewhat unclear. In transgenic mice expressing amyloid, higher amyloid was linked to higher neural excitability [44]. A recent study in transgenic mouse models of tau and amyloid suggests that amyloid is driving neuronal hyperactivity, but increased levels of tau lead to reduced neuronal activity [45]. However, these results are in conflict with previous results of the amyloid-independent association of tau-related susceptibility to hyperexcitability discussed above [41]. One possibility to reconcile the findings is that tau enhances amyloid-related neuronal hyperactivity at lower levels of amyloid, but reduces neuronal function at higher levels of amyloid. This stance would be in agreement with results from previous studies in humans reporting tau-PET but not amyloid-PET to be linked to fMRI-assessed hyperactivation [35] or FDG-PET hypermetabolism $[15,16]$. Furthermore, we observed FDG-PET hypermetabolism in the group of amyloid-negative/high-tau but not amyloid-positive/ low-tau suggesting that higher levels of tau in the presence of lower levels of amyloid are decisive for
FDG-PET hypermetabolism. As a third alternative, neuronal hyperexcitability may drive initial tau release, propagation, and spread [46, 47]. Future preclinical and intervention studies targeting amyloid or tau pathology will be instrumental in disentangling the causative relationship between primary AD pathologies and FDG-PET hypermetabolism.

Another major finding of our study was the association between FDG-PET hypermetabolism and lower memory performance suggesting that FDG-PET hypermetabolism may reflect pathologically altered FDG-PET levels that are detrimental rather than of compensatory nature. In previous studies including cognitively impaired elderly subjects, increased FDG-PET in the hippocampal formation was associated with poorer cognitive performance [48]. Moreover, reducing hippocampal hyperactivity by drug intervention improves cognition in MCI [49], where the same drug reduced taurelated neuronal hyperexcitability in a transgenic mouse model of AD [50]. Alternatively, higher neural activity may enhance tau spreading which in turn may lead to cognitive decline $[46,47]$. To test such a potentially mutually reinforcing chain of events would require longitudinal studies. With the caution that the current study does not allow for a causative interpretation, our 
findings suggest that local FDG-PET hypermetabolism in the presence of tau has no beneficial effect on cognition. We further caution that the MCI syndrome may have been also caused by other pathologies than amyloid and tau pathologies, especially in the MCI subjects with low amyloid. Alternative pathologies that have been linked to AD-like symptoms include cerebrovascular disease, aggregation of the transactive response DNA binding protein $43 \mathrm{kDa}$ (TDP-43), and alpha-synuclein [5154].

Several caveats need to be considered when interpreting the results of the current study. First, the current study is cross-sectional in nature. A longitudinal study will be informative to test the predictive value of tauand amyloid-PET for the subsequent changes in FDGPET and cognition. Second, the presence of the APOE $\varepsilon 4$ allele has been previously shown to be associated with glucose hypermetabolism [6] and thus may provide a confounding variable. However, a post hoc analysis showed that the observed interaction remained significant even when controlling for APOE genotype, suggesting that any association between APOE and tau pathology did not explain the current results. Third, although FDG-PET is commonly interpreted to reflect neural activity, it is possible that FDG-PET also reflects glial activity. For example, microglia activation is increased in relation to tau and amyloid pathology and can be associated with FDG-PET hypermetabolism as suggested by findings in mice [55]. However, our results on FDG-PET show parallels with the findings on restingstate and task-evoked fMRI BOLD signal which is less likely to reflect glia activity, discounting the possibility of glia activation as a major source of PET. Fourth, we did not apply partial volume correction to FDG-PET. We did so deliberately in order to avoid that FDG-PET hypermetabolism may occur due to the correction procedure. Here, we observed increased FDG-PET despite not correcting, supporting the view that a true increase in FDG-PET can be observed as a function of tau and amyloid pathology.

\section{Conclusions}

We found that FDG-PET hypermetabolism occurs as a function of increased tau-PET in the presence of low amyloid-PET, and is associated with worse cognitive performance. Our results have implications for clinical trials, where FDG-PET is often used as an outcome parameter [56]. Given the non-linear changes of FDGPET as a function of tau and amyloid pathology, a beneficial drug effect on FDG-PET may not always translate into a reduction in the decline of FDG-PET, but could also be a reduction of the detrimental increase in FDGPET. Clearly, our results call for a more sophisticated model of FDG-PET changes in the course of AD, taking both amyloid- and tau-PET into account.

\section{Supplementary information}

Supplementary information accompanies this paper at https://doi.org/10. 1186/s13195-020-00702-6.

Additional file 1: Figure S1. Regional interactions between amyloidand tau-PET on FDG-PET metabolism in MCl. Table S1. Areas showing significant voxel-wise effect of amyloid-PET and tau-PET on FDG-PET in $\mathrm{MCl}$.

\section{Abbreviations}

AD: Alzheimer's disease; ADNI: Alzheimer's Disease Neuroimaging Initiative; APOE: Apolipoprotein E; A : Beta-amyloid; CN: Cognitively normal; CSF: Cerebrospinal fluid; FDG: Fluorodeoxyglucose; GM: Gray matter; MCl: Mild cognitive impairment; MMSE: Mini-Mental State Examination; MRI: Magnetic resonance imaging; PET: Positron emission tomography; SUVR: Standard uptake value ratio; WM: White matter

\section{Acknowledgements}

Data used in the preparation of this article were obtained from the Alzheimer's Disease Neuroimaging Initiative (ADNI) database (adni.loni.usc. edu). As such, the investigators within the ADNI contributed to the design and implementation of ADNI and/or provided data but did not participate in the analysis or writing of this report. A complete list of ADNI investigators can be found at https://adni.loni.usc.edu/wp-content/uploads/how_to_ apply/ADNI_Acknowledgement_List.pdf.

\section{Authors' contributions}

AR conducted the analyses and wrote the manuscript, NF and JN provided critical review of the manuscript, and ME designed the study, interpreted the results, and wrote the manuscript. The authors read and approved the final manuscript.

\section{Funding}

The work was supported by the LMUexcellent Initiative (to ME) and DFG (German Research Foundation, INST 409/193-1 FUGG). ADNI data collection and sharing for this project was funded by the ADNI (National Institutes of Health Grant U01 AG024904) and DOD ADNI (Department of Defense award number W81XWH-12-2-0012). ADNI is funded by the National Institute on Aging, the National Institute of Biomedical Imaging, and Bioengineering, and through contributions from the following: AbbVie, Alzheimer's Association; Alzheimer's Drug Discovery Foundation; Araclon Biotech; BioClinica, Inc.; Biogen; Bristol-Myers Squibb Company; CereSpir, Inc.; Cogstate; Eisai Inc.; Elan Pharmaceuticals, Inc.; Eli Lilly and Company; Eurolmmun; F. Hoffmann-La Roche Ltd. and its affiliated company Genentech, Inc.; Fujirebio; GE Healthcare; IXICO Ltd.; Janssen Alzheimer Immunotherapy Research \& Development, LLC.; Johnson \& Johnson Pharmaceutical Research \& Development LLC.; Lumosity; Lundbeck; Merck \& Co., Inc.; Meso Scale Diagnostics, LLC.; NeuroRx Research; Neurotrack Technologies; Novartis Pharmaceuticals Corporation; Pfizer Inc.; Piramal Imaging; Servier; Takeda Pharmaceutical Company; and Transition Therapeutics. The Canadian Institutes of Health Research is providing funds to support ADNI clinical sites in Canada. Private sector contributions are facilitated by the Foundation for the National Institutes of Health (www.fnih.org). Open Access funding enabled and organized by Projekt DEAL.

\section{Availability of data and materials}

All neuroimaging and neuropsychology data that were used in this study are available online at the ADNI data repository (adni.loni.usc.edu).

Ethics approval and consent to participate

Ethical approval was obtained by the ADNI investigators, and all study participants provided written informed consent.

Consent for publication

Not applicable. 


\section{Competing interests}

The authors declare that they have no competing interests.

\section{Received: 15 April 2020 Accepted: 5 October 2020} Published online: 19 October 2020

\section{References}

1. Schwartz WJ, Smith CB, Davidsen L, Savaki H, Sokoloff L, Mata M, et al Metabolic mapping of functional activity in the hypothalamoneurohypophysial system of the rat. Science. 1979;205(4407):723.

2. Anchisi D, Borroni B, Franceschi M, Kerrouche N, Kalbe E, Beuthien-Beumann $B$, et al. Heterogeneity of brain glucose metabolism in mild cognitive impairment and clinical progression to Alzheimer disease. JAMA Neurol. 2005;62(11):1728-33.

3. Small GW, Mazziotta JC, Collins MT, Baxter LR, Phelps ME, Mandelkern MA et al. Apolipoprotein E type 4 allele and cerebral glucose metabolism in relatives at risk for familial Alzheimer disease. JAMA. 1995:273(12):942-7.

4. $\mathrm{Oh} \mathrm{H}$, Habeck $C$, Madison $C$, Jagust W. Covarying alterations in $A \beta$ deposition, glucose metabolism, and gray matter volume in cognitively normal elderly. Human brain mapping. 2014;35(1):https://doi.org/10.1002/ hbm.22173.

5. Benzinger TLS, Blazey T, Jack CR, Koeppe RA, Su Y, Xiong C, et al. Regional variability of imaging biomarkers in autosomal dominant Alzheimer's disease. Proc Natl Acad Sci U S A. 2013:110(47):E4502-E9.

6. Yi D, Lee DY, Sohn BK, Choe YM, Seo EH, Byun MS, et al. Beta-amyloid associated differential effects of APOE $\varepsilon 4$ on brain metabolism in cognitively normal elderly. Am J Geriatr Psychiatry. 2014;22(10):961-70.

7. Ashraf A, Fan Z, Brooks DJ, Edison P. Cortical hypermetabolism in MCl subjects: a compensatory mechanism? Eur J Nucl Med Mol Imaging. 2015; 42(3):447-58.

8. Edison P, Archer HA, Hinz R, Hammers A, Pavese N, Tai YF, et al. Amyloid, hypometabolism, and cognition in Alzheimer disease: an [11C]PIB and [18F]FDG PET study. Neurology. 2007;68(7):501-8.

9. Ewers M, Insel P, Jagust WJ, Shaw L, Trojanowski JJ, Aisen P, et al. CSF biomarker and PIB-PET-derived beta-amyloid signature predicts metabolic, gray matter, and cognitive changes in nondemented subjects. Cereb Cortex. 2012:22(9):1993-2004.

10. Li Y, Rinne JO, Mosconi L, Pirraglia E, Rusinek H, DeSanti S, et al. Regional analysis of FDG and PIB-PET images in normal aging, mild cognitive impairment, and Alzheimer's disease. Eur J Nucl Med Mol Imaging. 2008; 35(12):2169-81.

11. Rabinovici GD, Jagust WJ, Furst AJ, Ogar JM, Racine CA, Mormino EC, et al. Abeta amyloid and glucose metabolism in three variants of primary progressive aphasia. Ann Neurol. 2008:64(4):388-401.

12. Whitwell JL, Graff-Radford J, Tosakulwong N, Weigand SD, Machulda MM, Senjem ML, et al. Imaging correlations of tau, amyloid, metabolism, and atrophy in typical and atypical Alzheimer's disease. Alzheimers Dement. 2018;14(8):1005-14.

13. Ossenkoppele R, Schonhaut DR, Schöll M, Lockhart SN, Ayakta N, Baker SL, et al. Tau PET patterns mirror clinical and neuroanatomical variability in Alzheimer's disease. Brain. 2016;139(5):1551-67.

14. Dronse J, Fliessbach K, Bischof GN, von Reutern B, Faber J, Hammes J, et al. In vivo patterns of tau pathology, amyloid-beta burden, and neuronal dysfunction in clinical variants of Alzheimer's disease. J Alzheimers Dis. 2017; 55(2):465-71.

15. Adams JN, Lockhart SN, Li L, Jagust WJ. Relationships between tau and glucose metabolism reflect Alzheimer's disease pathology in cognitively normal older adults. Cerebral Cortex. 2018:bhy078-bhy.

16. Hanseeuw BJ, Betensky RA, Schultz AP, Papp KV, Mormino EC, Sepulcre J, et al. Fluorodeoxyglucose metabolism associated with tau-amyloid interaction predicts memory decline. Ann Neurol. 2017;81(4):583-96.

17. Weigand AJ, Bangen KJ, Thomas KR, Delano-Wood L, Gilbert PE, Brickman $\mathrm{AM}$, et al. Is tau in the absence of amyloid on the Alzheimer's continuum? a study of discordant PET positivity. Brain Commun. 2020;2(1):fcz046.

18. Franzmeier N, Duering M, Weiner M, Dichgans M, Ewers M, For the Alzheimer's Disease Neuroimaging I. Left frontal cortex connectivity underlies cognitive reserve in prodromal Alzheimer disease. Neurology. 2017:88(11):1054-1061.

19. Petersen RC, Aisen PS, Beckett LA, Donohue MC, Gamst AC, Harvey DJ, et al. Alzheimer's Disease Neuroimaging Initiative (ADNI): clinical characterization. Neurology. 2010;74(3):201-9.
20. Hansson O, Seibyl J, Stomrud E, Zetterberg H, Trojanowski JQ, Bittner T, et al. CSF biomarkers of Alzheimer's disease concord with amyloid- $\beta$ PET and predict clinical progression: a study of fully automated immunoassays in BioFINDER and ADNI cohorts. Alzheimer's \& Dementia: The Journal of the Alzheimer's Association.

21. Jagust WJ, Landau SM, Koeppe RA, Reiman EM, Chen K, Mathis CA, et al. The ADNI PET core: 2015. Alzheimer's Dementia. 2015;11(7):757-71.

22. Lowe VJ, Curran G, Fang P, Liesinger AM, Josephs KA, Parisi JE, et al. An autoradiographic evaluation of AV-1451 Tau PET in dementia. Acta Neuropathologica Communications. 2016;4:58.

23. Landau SM, Mintun MA, Joshi AD, Koeppe RA, Petersen RC, Aisen PS, et al Amyloid deposition, hypometabolism, and longitudinal cognitive decline. Ann Neurol. 2012:72(4):578-86

24. Crane PK, Carle A, Gibbons LE, Insel P, Mackin RS, Gross A, et al. Development and assessment of a composite score for memory in the Alzheimer's Disease Neuroimaging Initiative (ADNI). Brain Imaging Behavior. 2012;6(4):502-16.

25. Mathotaarachchi S, Wang S, Shin M, Pascoal TA, Benedet AL, Kang MS, et al. VoxelStats: a MATLAB package for multi-modal voxel-wise brain image analysis. Front Neuroinformatics. 2016;10:20.

26. Worsley KJ, Marrett S, Neelin P, Vandal AC, Friston KJ, Evans AC. A unified statistical approach for determining significant signals in images of cerebral activation. Hum Brain Mapp. 1996;4(1):58-73.

27. Cook RD. Detection of influential observation in linear regression. Technometrics. 1977;19(1):15-8.

28. Merlo S, Spampinato SF, Sortino MA. Early compensatory responses against neuronal injury: a new therapeutic window of opportunity for Alzheimer's disease? CNS Neurosci Ther. 2019:25(1):5-13.

29. Cohen AD, Price JC, Weissfeld LA, James J, Rosario BL, Bi W, et al. Basal cerebral metabolism may modulate the cognitive effects of $A \beta$ in mild cognitive impairment: an example of brain reserve. J Neurosci. 2009;29(47): 14770-8.

30. Putcha D, Brickhouse M, O'Keefe K, Sullivan C, Rentz D, Marshall G, et al. Hippocampal hyperactivation associated with cortical thinning in Alzheimer's disease signature regions in non-demented elderly adults. $J$ Neurosci. 2011:31(48):17680-8.

31. Clement F, Belleville S. Effect of disease severity on neural compensation of item and associative recognition in mild cognitive impairment. J Alzheimers Dis. 2012;29(1):109-23.

32. Quiroz YT, Budson AE, Celone K, Ruiz A, Newmark R, Castrillon G, et al. Hippocampal hyperactivation in presymptomatic familial Alzheimer's disease. Ann Neurol. 2010;68(6):865-75.

33. Dickerson BC, Salat DH, Greve DN, Chua EF, Rand-Giovannetti E, Rentz DM, et al. Increased hippocampal activation in mild cognitive impairment compared to normal aging and AD. Neurology. 2005:65(3):404-11.

34. Marks SM, Lockhart SN, Baker SL, Jagust WJ. Tau and beta-amyloid are associated with medial temporal lobe structure, function, and memory encoding in normal aging. J Neurosci. 2017;37(12):3192-201.

35. Huijbers W, Schultz AP, Papp KV, LaPoint MR, Hanseeuw B, Chhatwal JP, et al. Tau accumulation in clinically normal older adults is associated with hippocampal hyperactivity. J Neurosci. 2019;39(3):548-56.

36. Schultz AP, Chhatwal JP, Hedden T, Mormino EC, Hanseeuw BJ, Sepulcre J, et al. Phases of hyperconnectivity and hypoconnectivity in the default mode and salience networks track with amyloid and tau in clinically normal individuals. J Neurosci. 2017:37(16):4323-31.

37. Crary JF, Trojanowski JQ, Schneider JA, Abisambra JF, Abner EL, Alafuzoff I, et al. Primary age-related tauopathy (PART): a common pathology associated with human aging. Acta Neuropathol. 2014;128(6):755-66.

38. Bell WR, An Y, Kageyama Y, English C, Rudow GL, Pletnikova O, et al. Neuropathologic, genetic, and longitudinal cognitive profiles in primary age-related tauopathy (PART) and Alzheimer's disease. Alzheimers Dement. 2019;15(1):8-16.

39. Duyckaerts C, Braak H, Brion JP, Buee L, Del Tredici K, Goedert M, et al. PART is part of Alzheimer disease. Acta Neuropathol. 2015;129(5):749-56.

40. Bright J, Hussain S, Dang V, Wright S, Cooper B, Byun T, et al. Human secreted tau increases amyloid-beta production. Neurobiol Aging. 2015; 36(2):693-709.

41. Roberson ED, Halabisky B, Yoo JW, Yao J, Chin J, Yan F, et al. Amyloid- $\beta$ / Fyn-induced synaptic, network, and cognitive impairments depend on tau levels in multiple mouse models of Alzheimer's disease. J Neurosci. 2011; $31(2): 700$. 
42. Das M, Maeda S, Hu B, Yu GQ, Guo W, Lopez I, et al. Neuronal levels and sequence of tau modulate the power of brain rhythms. Neurobiol Dis. 2018; 117:181-8.

43. Levenga J, Krishnamurthy P, Rajamohamedsait H, Wong H, Franke TF, Cain $P$, et al. Tau pathology induces loss of GABAergic interneurons leading to altered synaptic plasticity and behavioral impairments. Acta Neuropathologica Communications. 2013;1(1):34.

44. Palop JJ, Chin J, Roberson ED, Wang J, Thwin MT, Bien-Ly N, et al. Aberrant excitatory neuronal activity and compensatory remodeling of inhibitory hippocampal circuits in mouse models of Alzheimer's disease. Neuron. 2007: 55(5):697-711.

45. Busche MA, Wegmann S, Dujardin S, Commins C, Schiantarelli J, Klickstein N, et al. Tau impairs neural circuits, dominating amyloid- $\beta$ effects, in Alzheimer models in vivo. Nat Neurosci. 2019;22(1):57-64.

46. Pooler AM, Phillips EC, Lau DHW, Noble W. Hanger DP. Physiological release of endogenous tau is stimulated by neuronal activity. EMBO Rep. 2013;14(4): 389-94.

47. Wu JW, Hussaini SA, Bastille IM, Rodriguez GA, Mrejeru A, Rilett K, et al. Neuronal activity enhances tau propagation and tau pathology in vivo. Nat Neurosci. 2016;19(8):1085-92.

48. Apostolova I, Lange C, Maurer A, Suppa P, Spies L, Grothe MJ, et al. Hypermetabolism in the hippocampal formation of cognitively impaired patients indicates detrimental maladaptation. Neurobiol Aging. 2018;65:41-50.

49. Bakker A, Krauss Gregory L, Albert Marilyn S, Speck Caroline L, Jones Lauren R, Stark Craig E, et al. Reduction of hippocampal hyperactivity improves cognition in amnestic mild cognitive impairment. Neuron. 2012;74(3):467-74.

50. Heneka MT, Carson MJ, El Khoury J, Landreth GE, Brosseron F, Feinstein DL, et al. Neuroinflammation in Alzheimer's disease. Lancet Neurol. 2015:14(4): 388-405.

51. Abner EL, Kryscio RJ, Schmitt FA, Fardo DW, Moga DC, Ighodaro ET, et al. Outcomes after diagnosis of mild cognitive impairment in a large autopsy series. Ann Neurol. 2017;81(4):549-59.

52. Schneider JA, Arvanitakis Z, Leurgans SE, Bennett DA. The neuropathology of probable Alzheimer disease and mild cognitive impairment. Ann Neurol. 2009;66(2):200-8.

53. Rahimi J, Kovacs GG. Prevalence of mixed pathologies in the aging brain. Alzheimers Res Ther. 2014;6(9):82

54. Nelson PT, Trojanowski JQ, Abner EL, Al-Janabi OM, Jicha GA, Schmitt FA, et al. "New old pathologies": AD, PART, and cerebral age-related TDP-43 with sclerosis (CARTS). J Neuropathol Exp Neurol. 2016;75(6):482-98.

55. Brendel M, Focke C, Blume T, Peters F, Deussing M, Probst F, et al. Time courses of cortical glucose metabolism and microglial activity across the life span of wild-type mice: a PET study. J Nuclear Med. 2017;58(12):1984-90

56. Herholz K, Boecker H, Nemeth I, Dunn G. FDG PET in dementia multicenter studies and clinical trials. Clin Translational Imaging. 2013;1(4):261-70.

\section{Publisher's Note}

Springer Nature remains neutral with regard to jurisdictional claims in published maps and institutional affiliations.

Ready to submit your research? Choose BMC and benefit from:

- fast, convenient online submission

- thorough peer review by experienced researchers in your field

- rapid publication on acceptance

- support for research data, including large and complex data types

- gold Open Access which fosters wider collaboration and increased citations

- maximum visibility for your research: over $100 \mathrm{M}$ website views per year

At BMC, research is always in progress.

Learn more biomedcentral.com/submissions 